\title{
ORCHID DIVERSITY IN ANTHROPOGENIC-INDUCED DEGRADED TROPICAL RAINFOREST, AN EXTRAPOLATION TOWARDS CONSERVATION
}

\author{
Edward E. Besi, Dome Nikong, Muskhazli Mustafa \& Rusea Go* \\ Department of Biology, Faculty of Science, Universiti Putra Malaysia, 43400 Serdang, \\ Selangor Darul Ehsan, Malaysia \\ *Corresponding author: rusea@upm.edu.my
}

\begin{abstract}
The uncontrolled logging in Peninsular Malaysia and the resulting mudslides in the lowland areas have been perilous, not to just humans, but also to another biodiversity, including the wild orchids. Their survival in these highly depleted areas is being overlooked due to the inaccessible and harsh environment. This paper reports on the rescue of orchids at risk from the disturbed forests for ex-situ conservation, the identification of the diversity of orchids and the evaluation of the influence of micro-climatic changes induced by clear-cut logging towards the resilience of orchids in the flood-disturbed secondary forests and logged forests in Terengganu and Kelantan, located at the central region of Peninsular Malaysia, where the forest destruction by logging activities has been extensive. 109 orchid species belonging to 40 genera were collected from the disturbed areas. The diversity and data analyses show that the disturbed secondary forests had a higher orchid density ( 0.0133 plants/ $\left.\mathrm{m}^{2}\right)$ than the logged sites $\left(0.0040\right.$ plants $\left./ \mathrm{m}^{2}\right)$ as the habitat conditions were more dependable. Nevertheless, the logged forests harboured a higher diversity of orchids $(\mathrm{H}=4.50$ and $\mathrm{D}=0.99)$ of which $97.9 \%$ were epiphytes. Eleven rare species were found along with six species endemic to Peninsular Malaysia, with two species new to science. The results highlighted the factors that allow the orchids to flourish or suffer in the disturbed forests. The logged forests had a higher ambient temperature and lower moisture level than the mud flood-disturbed and canopy-covered secondary forests. Apart from the extensive ground vegetation due to logs dragging extraction, low soil moisture and absence of leaf litter were believed to be the major attributes causing the low abundance of terrestrial orchids. The high abundance and diversity of epiphytic orchids and the large difference of their densities between the logged sites were influenced by the densities of fallen trees hosting orchid(s), disturbanceinduced dryness stresses, durations of exposure to the anthropogenic-induced disturbance, and less favourable soil conditions for the terrestrial orchids.

KEY WORDS: conservation, diversity, dryness stress, ecology, epiphyte, logged forest, mudslides, Orchidaceae
\end{abstract}

Introduction. Kuala Koh, Kelantan and Tasek Kenyir, Terengganu are the largest and commonly visited parts of the Peninsular Malaysia's National Park. Both are located in the northeast states of Peninsular Malaysia. Tasek Kenyir is an artificial lake formed by Kenyir Dam or the Sultan Mahmud Hydro-Electric Power Station, which took 15 years to be constructed and fully operate in 1978. Both areas are gazetted as a protected forest reserve under the Taman Negara (Kelantan) Enactment [En. 14 of 1938] and the Taman Negara (Terengganu) Enactment [En. 6 of 1939]. These Acts are independent from The National Parks Act 1980. These areas have many geological and biological attractions (Hairul et al. 2016), apart from being home for the aboriginal tribes of Semaq (Hulu Terengganu) and Bateq (Kuala Koh, Kelantan). The tribal people forage the forest for food and medicines apart from hunting and fishing for their daily necessities (Ramle 1993, Fatanah 2009, Ramle et al. 2014, Abdullah et al. 2017). Topographically, the Kuala Koh and its adjacent area consist of riverine, largely hill and lowland dipterocarp forests. The shaded and humid environment encourages the growth of mosses on the trees, creating niches suitable for moisture-loving epiphytes. In over logged forest, epiphytic orchids are also found growing in abundance on the ground and on rocks, and a few relying on dead debris living as mycoheterotrophs. However, both terrestrial and epiphytic orchids have experienced population declined mainly because of habitat degradation and timber extraction 
process, which have caused many species to near extinction (Larson 1992, Rauh 1992, Dimmitt 2000, Mondragon \& Calvo-Irabien 2006).

The Orchidaceae is a speciose family of considerable significance in horticulture (Hew et al. 1997), and traditional medicine, with many species in various genera having been reported to have therapeutic properties (Pant 2013). Unfortunately, many orchids are naturally rare, endangered, or vulnerable, for climate and habitat changes that are often anthropogenic, and from over-collection for horticulture and medicine. Some 972 species of orchids in 159 genera have been recorded in Peninsular Malaysia (Go \& Hamzah 2008, Go et al. 2010, Kiew et al. 2010, Ong et al. 2017). About twenty percent of them are endemic to Peninsular Malaysia (Seidenfaden \& Wood 1992, Turner 1995, Ong et al. 2011). Until recently, a total of 245 orchid species were collected from Terengganu and 223 orchid species from Kelantan as documented in SING Herbarium (2018, January), Swiss Orchid Foundation at the Herbarium Jany Renz (2017, July), National Herbarium of the Netherlands (NHN) (2017, July), Turner (1995), and Jaafar et al. (2007).

In recent years, human activities in Kuala Koh and around Tasek Kenyir have directly threatened the survivability of biodiversity including orchids. Kuala Koh and its vicinities are now blatantly known to have the highest rate of deforestation in Peninsular Malaysia. A large tract of forest near the National Park entrance has been cleared for farming, mining and logging (Tuck-Po 2000, Lye 2005, Hairul et al. 2016). Forest clearance has spread extensively to Tasek Kenyir area. The clay exposed cleared land caused surface water run-off and land erosion due to lack of retention from grasses, trees, and shrubs had caused the 2014 tragic flash floods and mudslides in the lowland areas of Terengganu and Kelantan. Based on our empirical observations, the uncontrolled and unsustainable timber production might have concurrently shoved the epiphytic orchid flora towards local extinction. The canopy disruptions caused by timber extractions have greatly modified the temperature, humidity, and light conditions, causing unfavourable habitat for the shadeloving species (Gradstein 2008, Benítez et al. 2015). Thus, the extremely harsh conditions in the logging sites (logged forests) where full exposure to the sun, water stress, and nutrient-poor soil due to the eroded topsoil and nutrient were detrimental for the orchid survival (Wan Mohd Shukri et al. 2007). Coupled with rampant forests destruction in the surrounding areas, the mudslides have also negatively affected the ecosystem balance, especially for the forest floor biodiversity including terrestrial orchids and waterways.

Deforestation and the global climate change have been largely ignored by the authority due to lack of assessing personnel willing to work in dangerous fragile logging sites, thus a stumbling block for conservation efforts to protect forest biodiversity including orchids in Malaysia. Because of their ecological, economical and pharmaceutical importance, an exhaustive evaluation on the diversity and ecology of orchids in the degraded forest areas is crucial for setting its conservation priorities. Hence, this paper focuses on reporting the diversity and distribution of orchids at risk in the degraded forests by uncovering the significance of ecological alterations that are negatively affecting their survival and to provide inference for ex-situ conservation plans.

\section{Materials and Methods}

Selection of field sampling localities and ecosystems.The study areas were classified based on the type of disturbance: disturbed secondary forests (DSFs) damaged by mudslides, and logging sites. Observations and botanical collection trips were carried out in the disturbed forests of Terengganu and Kelantan regions from November 2016 until May 2018 (Fig. 1). The areas covered in this study were DSFs in Kuala Koh, Gua Musang, Kelantan (94.4-129.9 m a.s.1.) and Air Canal, Tanah Merah, Kelantan (44 m a.s.1.); and logging sites in Tasek Kenyir, Terengganu (98.8-330.4 $\mathrm{m}$ a.s.1.). The disturbed forests were selected based on whether logging was imminent, currently actively logged, and the recent deadly mudslides repercussion. Eight short line-transect plots of $20 \mathrm{~m} \times 5 \mathrm{~m}$ with a total area of $800 \mathrm{~m}^{2}$ were established in Kuala Koh. Meanwhile, two line-transect plots of $25 \mathrm{~m} \times 5 \mathrm{~m}$ were created in disturbed lowland secondary forest at Air Canal with the total area of $250 \mathrm{~m}^{2}$. Three logging sites in the Tasek Kenyir area were selected as study sites. These sites were located using Google Maps and also based on an earlier preliminary empirical study. Site 1 and Site 2 of active logging were located in Gawi, and Site 3 was located in Petuang, with a total area studied 


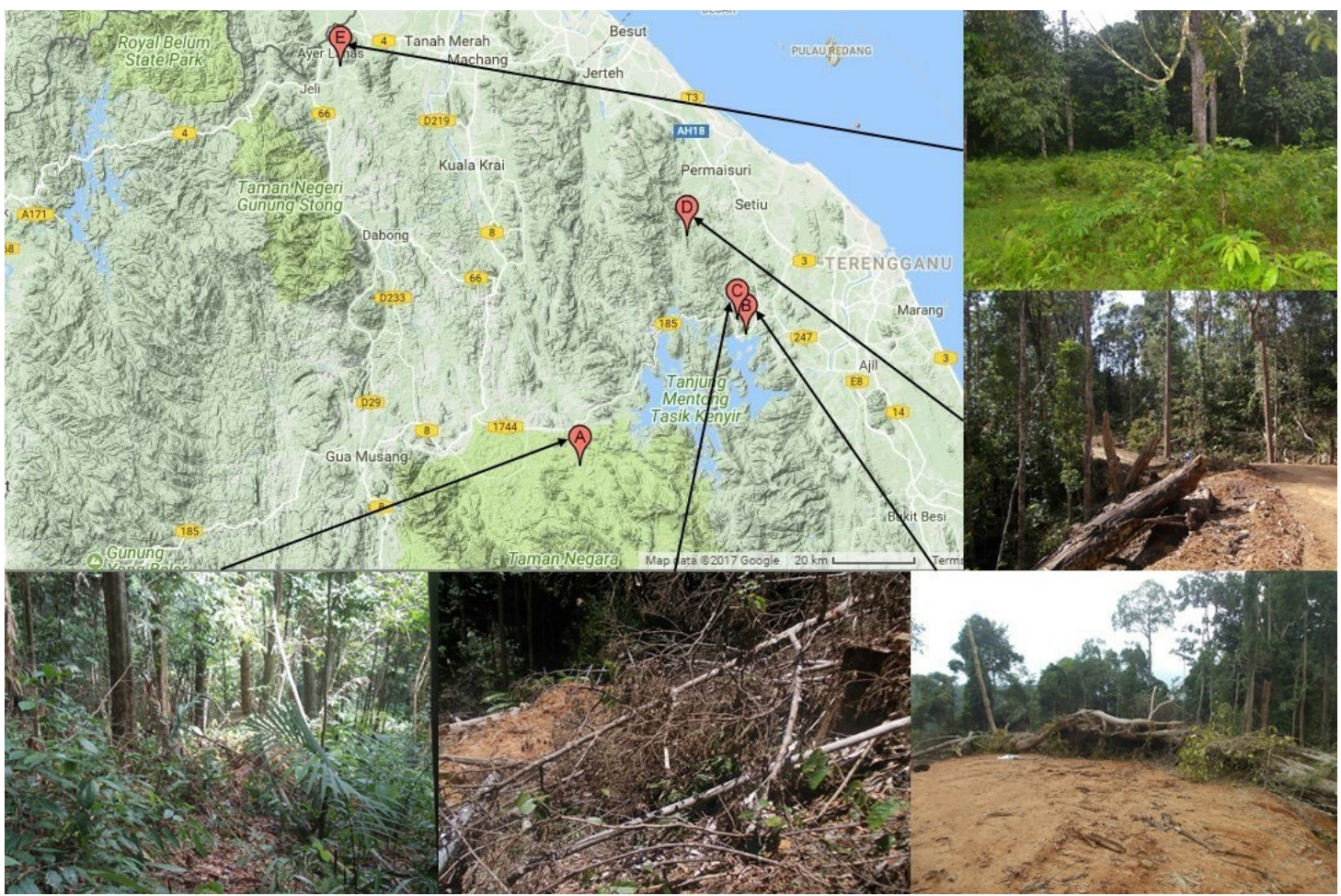

Figure 1. Coordinates of each studied site recorded in disturbed forests of Terengganu and Kelantan regions. A. Kuala Koh area (DSFs Site 1). B. Gawi area (Logging Site 1). C. Gawi area (Logging Site 2). D. Petuang area (Logging Site 3). E. Tanah Merah area (DSFs Site 2). The map was adapted from http://www.geoplaner.com, a free web-based application that provides several GIS and GPS services.

of approximately $53,000 \mathrm{~m}^{2}$. The total area studied in the logging sites was calculated based on the distance traveled along the logging road in the logging site times the width of $20 \mathrm{~m}$ on each side of the road, and the orchids were mostly found on fallen trees.

Fieldwork and sample collection.- The systematic sampling involved choosing exploration sites based on the existence of disturbed forests in the Terengganu and Kelantan. The date and time of the sample collections were scheduled based on the type of disturbance (DSFs and logging site) and the accessibility (weighed more towards the accessing highly disturbed and risky logging site areas). Non-flowering orchids were transferred to an ex-situ facility in Setiu, Terengganu for further flowering monitoring to ascertain the species identity.

Sample processing and identification.- Identifiable samples (with floral structures) were preserved using standard herbarium technique after Bridson and Forman (2000) and the non-flowering ones were transplanted into the ex-situ nursery, as living collections, where they were nurtured until flowering usually within five to six months, depending on the species (Go et al. 2011). Reliable references and online databases were used in the identification process and evaluation of each species' distribution status: Seidenfaden \& Wood (1992), Turner (1995), Comber (2001), Ong et al. (2017), the Swiss Orchid Foundation at the Herbarium Jany Renz. (2017, July), the National Herbarium of the Netherlands (NHN) accessed through Browse Dutch Natural History Collections: BioPortal (Naturalis) (2017, July), and the World Checklist of Selected Plant Families (WCSP) (2018, December). Also, expert consultations were sought to make identifications.

Diversity analyses.- Shannon-Wiener Diversity Index (H) and Simpson's Diversity Index (D) were used to determine the species richness and evenness in both 
studied areas. The Shannon index in real ecosystems ranges between 1.5 and 3.5 (MacDonald 2003). The $\mathrm{H}$ value rarely surpasses 4.5 , and if it does increase, the increment is small due to the logarithmic element in the function (Margalef 1972). The greater the value, the higher the diversity. The $\mathrm{D}$ value ranges between 0 and $1 ; 1$ represents infinite diversity and 0 , no diversity. The closer the value is to 1 , the higher the diversity. However, these methods could not tell which factors contributed more to the value, hence, Evenness (E) was used to determine how close in numbers each species in each studied site (Help 1974). The E value is constrained between 0 and 1 . The lower $\mathrm{E}$ value means the less evenness in the communities between the species with presence of dominant species, and the lower the diversity. The formula is derived according to Pielou's evenness index (Pielou 1966). The frequency of occurrence of each species was determined based on the number of times that particular species occurs or sighted at a specified sample plot.

In-situ measurement of ecological data-- Minimum (min) and maximum (max) values for four ecological parameters were recorded from morning (10:00 a.m.) to afternoon (4:00 p.m.) time in the studied disturbed forests of Terengganu and Kelantan: temperature $\left({ }^{\circ} \mathrm{C}\right)$, air relative humidity (ARH) (1-100\%), soil moisture (SM) (1-10\%), and light intensity (Lux or 1x).

Data analyses.- All the data were recorded in a spreadsheet, and the following parameters were computed as follows: 1) Density of the orchid species within a plot (plants $\left./ \mathrm{m}^{2}\right)$ : Number of orchid species within the plot (plants) / Total area of the plot $\left(\mathrm{m}^{2}\right)$; 2) Density of fallen trees hosting orchid(s) within a plot (plants $/ \mathrm{m}^{2}$ ): Number of fallen trees in the plot hosting orchids (plants) / Total area of the plot $\left(\mathrm{m}^{2}\right)$; and 3) Relative abundance of orchid (\%Ao): [Number of clump or population of a particular orchid species within the plot / Total number of all orchid clumps or populations of the plot] $\times 100$.

Statistical analyses.- The ecological data were analysed using IBM SPSS version 19. Non-parametric Kruskal-Wallis one-way analysis of variance (ANOVA) with multiple pairwise comparisons $(p<0.05)$ was used to determine the significant relationship of the ecological parameters between the different studied sites with distinctively different disturbance types. The significant difference of mean between groups was determined by comparing directly between the min values recorded for DSFs with min values recorded for logging sites and were also evaluated accordingly for the max values. The Pearson's correlation coefficient was used to measure the linear relationship between the orchid abundance and their host trees; Density of the orchid species within a plot (plants $/ \mathrm{m}^{2}$ ) and Density of fallen trees hosting orchid(s) within a plot (plants $/ \mathrm{m}^{2}$ ).

\section{Results}

Abundance and distribution pattern.- 132 orchid specimens of 109 orchid species belonging to 40 genera were collected from the disturbed forests (Table 1). Of these, 116 orchid specimens belonging to 96 species were recorded from the Tasek Kenyir logging sites, whereas, 16 specimens of 14 species were recorded from the DSFs. $93.3 \%$ of the total number of orchid species collected were epiphytes and 3.7\% were terrestrials. Based on Table 1, orchid species collected from the disturbed forests were largely Epidendroideae subfamily with 107 species $(98.2 \%)$, and only one species was from Orchidoideae $(0.9 \%)$ and Apostasioideae (0.9\%) subfamilies. Species of the genera Bulbophyllum Thouars and Dendrobium Sw. were found to be the most abundant orchids living in these disturbed forests. The most abundant species found in the logging sites were Dendrobium crumenatum Sw. (3.2\%) and Grammatophyllum speciosum Blume (2.5\%), whereas Corymborkis veratrifolia (Reinw.) Blume (3.2\%) was abundant in the DSFs. A total of 87 species $(79.8 \%)$ found in the disturbed forests were growing with a sympodial habit. The remaining 22 species (20.2\%) were monopodial, of which, 12 species (11\%) were climbers.

Rareness and endemism.- Ninety-two orchid species (84.4\%) collected were common and widespread. Eleven orchid species $(10.1 \%)$ were rare (recorded with a very narrow endemic range or less than five locations of occupancy) or uncommon (more abundant than a rare species) which were previously recorded with small distribution area. Six orchid species (5.5\%) endemic to Peninsular Malaysia were also discovered; namely, Bulbophyllum linearifolium 
TABLE 1. List of orchid species collected from the disturbed forests of Terengganu and Kelantan, and details on their habit, abundance and distribution status. Notes: \%Ao: Relative abundance of orchid; EP: Epiphyte; TR: Terrestrial; CM: Common; RR: Rare; NS: New Species; ED(PM): Endemic to Peninsular Malaysia.

\begin{tabular}{|c|c|c|c|c|}
\hline Subfamily/Genus & Species & $\%$ Ao & $\begin{array}{c}\text { Growth } \\
\text { Habit }\end{array}$ & $\begin{array}{c}\text { Distribution } \\
\text { Status }\end{array}$ \\
\hline \multicolumn{5}{|l|}{ Epidendroideae } \\
\hline Acriopsis & 1. Acriopsis liliifolia (J.Koenig) Seidenf. var. liliifolia & 1.3 & EP & $\mathrm{CM}$ \\
\hline \multirow[t]{2}{*}{ Adenoncos } & 2. Adenoncos major Ridl. & 0.6 & EP & $\mathrm{CM}$ \\
\hline & 3. Adenoncos vesiculosa Carr & 0.6 & EP & $\mathrm{CM}$ \\
\hline Aerides & 4. Aerides odorata Lour. & 1.3 & EP & $\mathrm{CM}$ \\
\hline \multirow[t]{3}{*}{ Agrostophyllum } & 5. Agrostophyllum glumaceum Hook.f. & 0.6 & EP & $\mathrm{CM}$ \\
\hline & 6. Agrostophyllum stipulatum subsp. bicuspidatum (J.J.Sm.) Schuit. & 0.6 & EP & $\mathrm{CM}$ \\
\hline & 7. Agrostophyllum stipulatum (Griff.) Schltr. subsp. stipulatum & 0.6 & EP & $\mathrm{CM}$ \\
\hline \multirow[t]{2}{*}{ Appendicula } & 8. Appendicula lucida Ridl. & 0.6 & EP & $\mathrm{CM}$ \\
\hline & 9. Appendicula uncata Ridl. subsp. uncata & 0.6 & EP & $E D(P M)$ \\
\hline \multirow[t]{2}{*}{ Bromheadia } & 10. Bromheadia alticola Ridl. & 0.6 & EP & $\mathrm{CM}$ \\
\hline & 11. Bromheadia petuangensis Rusea \& Besi $s p$. nov. & 0.6 & EP & NS \\
\hline \multirow[t]{21}{*}{ Bulbophyllum } & 12. Bulbophyllum armeniacum J.J.Sm. & 0.6 & EP & $\mathrm{CM}$ \\
\hline & 13. Bulbophyllum bakhuizenii Steenis & 0.6 & EP & $\mathrm{CM}$ \\
\hline & 14. Bulbophyllum biflorum Teijsm. \& Binn. & 0.6 & EP & $\mathrm{CM}$ \\
\hline & 15. Bulbophyllum biseriale Carr & 0.6 & EP & $\mathrm{CM}$ \\
\hline & 16. Bulbophyllum cf. caudatisepalum & 0.6 & EP & $\mathrm{CM}$ \\
\hline & 17. Bulbophyllum cf. corolliferum & 0.6 & EP & $\mathrm{CM}$ \\
\hline & 18. Bulbophyllum cf. flavescens & 0.6 & EP & $\mathrm{CM}$ \\
\hline & 19. Bulbophyllum corolliferum J.J.Sm. & 1.9 & EP & $\mathrm{CM}$ \\
\hline & 20. Bulbophyllum ecornutum (J.J.Sm.) J.J.Sm. subsp. ecornutum & 0.6 & EP & $\mathrm{RR}$ \\
\hline & 21. Bulbophyllum elevatopunctatum J.J.Sm. & 0.6 & EP & $\mathrm{RR}$ \\
\hline & 22. Bulbophyllum gracillimum (Rolfe) Rolfe & 0.6 & EP & $\mathrm{CM}$ \\
\hline & 23. Bulbophyllum lasiochilum E.C.Parish \& Rchb.f. & 0.6 & EP & $\mathrm{RR}$ \\
\hline & 24. Bulbophyllum limbatum Lindl. & 1.3 & EP & $\mathrm{RR}$ \\
\hline & 25. Bulbophyllum linearifolium King \& Pantl. & 0.6 & EP & $\mathrm{ED}(\mathrm{PM})$ \\
\hline & 26. Bulbophyllum macranthum Lindl. & 0.6 & EP & $\mathrm{CM}$ \\
\hline & 27. Bulbophyllum medusae (Lindl.) Rchb.f. & 0.6 & EP & $\mathrm{CM}$ \\
\hline & 28. Bulbophyllum patens King ex Hook.f. & 0.6 & EP & $\mathrm{CM}$ \\
\hline & 29. Bulbophyllum setuliferum J.J.Verm. \& Saw & 0.6 & EP & $\mathrm{ED}(\mathrm{PM})$ \\
\hline & 30. Bulbophyllum sp. (1) & 0.6 & EP & $\mathrm{CM}$ \\
\hline & 31. Bulbophyllum vaginatum (Lindl.) Rchb.f. & 1.3 & EP & $\mathrm{CM}$ \\
\hline & 32. Bulbophyllum vermiculare Hook.f. & 0.6 & EP & $\mathrm{CM}$ \\
\hline Calanthe & 33. Calanthe ceciliae Rchb.f. & 0.6 & TR & $\mathrm{CM}$ \\
\hline Callostylis & 34. Callostylis pulchella (Lindl.) S.C.Chen \& Z.H.Tsi & 0.6 & EP & $\mathrm{CM}$ \\
\hline \multirow[t]{2}{*}{ Campanulorchis } & 35. Campanulorchis leiophylla (Lindl.) Y.P.Ng \& P.J.Cribb & 0.6 & EP & $\mathrm{CM}$ \\
\hline & 36. Campanulorchis pellipes (Rchb.f. ex Hook.f.) Y.P.Ng \& P.J.Cribb & 1.3 & EP & $\mathrm{CM}$ \\
\hline
\end{tabular}




\begin{tabular}{|c|c|c|c|c|}
\hline Ceratostylis & 37. Ceratostylis pendula Hook.f. & 1.3 & EP & $\mathrm{CM}$ \\
\hline & 38. Ceratostylis subulata Blume & 0.6 & EP & $\mathrm{CM}$ \\
\hline \multirow[t]{3}{*}{ Cleisostoma } & 39. Cleisostoma discolor Lindl. & 0.6 & EP & $\mathrm{CM}$ \\
\hline & 40. Cleisostoma scortechinii (Hook.f.) Garay & 0.6 & EP & $\mathrm{CM}$ \\
\hline & 41. Cleisostoma sp. (1) & 0.6 & EP & $\mathrm{CM}$ \\
\hline \multirow[t]{3}{*}{ Coelogyne } & 42. Coelogyne cumingii Lindl. & 0.6 & EP & $\mathrm{CM}$ \\
\hline & 43. Coelogyne foerstermannii Rchb.f. & 1.3 & EP & $\mathrm{CM}$ \\
\hline & 44. Coelogyne tomentosa Lindl. & 0.6 & EP & $\mathrm{CM}$ \\
\hline Corymborkis & 45. Corymborkis veratrifolia (Reinw.) Blume & 3.2 & TR & $\mathrm{CM}$ \\
\hline \multirow[t]{2}{*}{ Cylindrolobus } & 46. Cylindrolobus mucronatus (Lindl.) Rauschert & 0.6 & EP & $\mathrm{CM}$ \\
\hline & 47. Cylindrolobus neglectus (Ridl.) J.J.Wood & 0.6 & EP & $\mathrm{CM}$ \\
\hline \multirow[t]{2}{*}{ Cymbidium } & 48. Cymbidium bicolor subsp. pubescens (Lindl.) Du Puy \& P.J.Cribb & 0.6 & EP & $\mathrm{CM}$ \\
\hline & 49. Cymbidium finlaysonianum Lindl. & 0.6 & EP & $\mathrm{CM}$ \\
\hline \multirow[t]{21}{*}{ Dendrobium } & 50. Dendrobium acerosum Lindl. & 1.3 & EP & $\mathrm{CM}$ \\
\hline & 51. Dendrobium angustifolium (Blume) Lindl. & 0.6 & EP & $\mathrm{CM}$ \\
\hline & 52. Dendrobium bancanum J.J.Sm. & 1.9 & EP & $\mathrm{CM}$ \\
\hline & 53. Dendrobium cf. linguella & 0.6 & EP & $\mathrm{CM}$ \\
\hline & 54. Dendrobium connatum (Blume) Lindl. var. connatum & 0.6 & EP & $\mathrm{CM}$ \\
\hline & 55. Dendrobium convexum (Blume) Lindl. & 1.9 & EP & $\mathrm{CM}$ \\
\hline & 56. Dendrobium crumenatum Sw. & 3.2 & EP & $\mathrm{CM}$ \\
\hline & 57. Dendrobium farmeri Paxton & 0.6 & EP & $\mathrm{CM}$ \\
\hline & 58. Dendrobium indivisum (Blume) Miq. var. indivisum & 1.3 & EP & $\mathrm{CM}$ \\
\hline & 59. Dendrobium indragiriense Schltr. & 0.6 & EP & $\mathrm{CM}$ \\
\hline & 60. Dendrobium kentrophyllum Hook.f. & 0.6 & EP & $\mathrm{CM}$ \\
\hline & 61. Dendrobium lamellatum (Blume) Lindl. & 0.6 & EP & RR \\
\hline & 62. Dendrobium leonis (Lindl.) Rchb.f. & 1.3 & EP & $\mathrm{CM}$ \\
\hline & 63. Dendrobium pachyphyllum (Kuntze) Bakh.f. & 1.3 & EP & $\mathrm{CM}$ \\
\hline & 64. Dendrobium plicatile Lindl. & 0.6 & EP & $\mathrm{CM}$ \\
\hline & 65. Dendrobium quadrilobatum Carr & 1.3 & EP & $\mathrm{RR}$ \\
\hline & 66. Dendrobium rhodostele Ridl. & 0.6 & EP & $\mathrm{CM}$ \\
\hline & 67. Dendrobium singaporense A.D.Hawkes \& A.H.Heller & 1.3 & EP & $\mathrm{CM}$ \\
\hline & 68. Dendrobium sp. (1) & 0.6 & EP & RR \\
\hline & 69. Dendrobium tortile Lindl. & 0.6 & EP & $\mathrm{CM}$ \\
\hline & 70. Dendrobium zebrinum J.J.Sm. & 0.6 & EP & $\mathrm{CM}$ \\
\hline Dendrochilum & 71. Dendrochilum pallidiflavens Blume var. pallidiflavens & 0.6 & EP & $\mathrm{CM}$ \\
\hline \multirow[t]{2}{*}{ Eria } & 72. Eria atrovinosa Carr & 1.3 & EP & $\mathrm{CM}$ \\
\hline & 73. Eria javanica (Sw.) Blume & 0.6 & EP & $\mathrm{CM}$ \\
\hline Grammatophyllum & 74. Grammatophyllum speciosum Blume & 2.5 & EP & $\mathrm{CM}$ \\
\hline Grosourdya & 75. Grosourdya cf. muscosa & 0.6 & EP & RR \\
\hline Liparis & 76. Liparis elegans Lindl. & 0.6 & EP & $\mathrm{CM}$ \\
\hline
\end{tabular}




\begin{tabular}{|c|c|c|c|c|}
\hline Micropera & 77. Micropera fuscolutea (Lindl.) Garay & 0.6 & EP & $\mathrm{RR}$ \\
\hline & 78. Micropera pallida (Roxb.) Lindl. & 0.6 & EP & $\mathrm{CM}$ \\
\hline \multirow[t]{2}{*}{ Mycaranthes } & 79. Mycaranthes latifolia Blume & 0.6 & EP & $\mathrm{CM}$ \\
\hline & 80. Mycaranthes pannea (Lindl.) S.C.Chen \& J.J.Wood & 1.3 & EP & $\mathrm{CM}$ \\
\hline \multirow[t]{3}{*}{ Oberonia } & 81. Oberonia brachystachys Lindl. & 0.6 & EP & $\mathrm{CM}$ \\
\hline & 82. Oberonia ciliolata Hook.f. & 0.6 & EP & $\mathrm{CM}$ \\
\hline & 83. Oberonia insectifera Hook.f. & 0.6 & EP & $\mathrm{CM}$ \\
\hline Oxystophyllum & 84. Oxystophyllum carnosum Blume & 1.9 & EP & $\mathrm{CM}$ \\
\hline Pholidota & 85. Pholidota articulata Lindl. & 0.6 & EP & $\mathrm{CM}$ \\
\hline Phreatia & 86. Phreatia plantaginifolia (J.Koenig) Ormerod & 0.6 & EP & $\mathrm{CM}$ \\
\hline \multirow[t]{3}{*}{ Pinalia } & 87. Pinalia domii Rusea \& Besi sp. nov. & 0.6 & EP & NS \\
\hline & 88. Pinalia floribunda (Lindl.) Kuntze & 1.9 & EP & $\mathrm{CM}$ \\
\hline & 89. Pinalia maingayi (Hook.f.) Kuntze & 0.6 & EP & $\mathrm{ED}(\mathrm{PM})$ \\
\hline \multirow[t]{2}{*}{ Pomatocalpa } & 90. Pomatocalpa diffusum Breda & 2.5 & EP & $\mathrm{CM}$ \\
\hline & 91. Pomatocalpa spicatum Breda, Kuhl \& Hasselt & 1.9 & EP & $\mathrm{CM}$ \\
\hline Renanthera & 92. Renanthera histrionica Rchb.f. & 0.6 & EP & $\mathrm{CM}$ \\
\hline Rhynchostylis & 93. Rhynchostylis retusa (L.) Blume & 1.9 & EP & $\mathrm{CM}$ \\
\hline Stichorkis & 94. Stichorkis gibbosa (Finet) J.J.Wood & 1.3 & EP & $\mathrm{CM}$ \\
\hline \multirow[t]{2}{*}{ Taeniophyllum } & 95. Taeniophyllum pusillum (Willd.) Seidenf. \& Ormerod & 0.6 & EP & $\mathrm{RR}$ \\
\hline & 96. Taeniophyllum sp. (1) & 0.6 & EP & $\mathrm{RR}$ \\
\hline Thecostele & 97. Thecostele alata (Roxb.) E.C.Parish \& Rchb.f. & 0.6 & EP & $\mathrm{CM}$ \\
\hline \multirow[t]{2}{*}{ Thelasis } & 98. Thelasis carinata Blume & 1.3 & EP & $\mathrm{CM}$ \\
\hline & 99. Thelasis pygmaea (Griff.) Lindl. & 0.6 & EP & $\mathrm{CM}$ \\
\hline \multirow[t]{7}{*}{ Thrixspermum } & $\begin{array}{l}\text { 100. Thrixspermum acuminatissimum (Blume) Rchb.f. subsp. } \\
\text { acuminatissimum }\end{array}$ & 1.3 & EP & $\mathrm{CM}$ \\
\hline & 101. Thrixspermum centipeda Lour. & 1.3 & EP & $\mathrm{CM}$ \\
\hline & 102. Thrixspermum cf. carnosum & 0.6 & EP & $\mathrm{CM}$ \\
\hline & 103. Thrixspermum clavatum (J.Koenig) Garay & 0.6 & EP & $\mathrm{CM}$ \\
\hline & 104. Thrixspermum sp. (1) & 0.6 & EP & $\mathrm{CM}$ \\
\hline & 105. Thrixspermum sp. (2) & 0.6 & EP & $\mathrm{CM}$ \\
\hline & 106. Thrixspermum trichoglottis (Hook.f.) Kuntze & 0.6 & EP & $\mathrm{CM}$ \\
\hline Trichotosia & 107. Trichotosia gracilis (Hook.f.) Kraenzl. & 0.6 & EP & $\mathrm{CM}$ \\
\hline \multicolumn{5}{|c|}{ Apostasioideae } \\
\hline Apostasia & 108. Apostasia nuda R.Br. & 1.9 & TR & $\mathrm{CM}$ \\
\hline \multicolumn{5}{|c|}{ Orchidoideae } \\
\hline Hetaeria & 109. Hetaeria oblongifolia Blume & 0.6 & TR & $\mathrm{CM}$ \\
\hline
\end{tabular}

King \& Pantl., B. setuliferum J.J.Verm. \& Saw, Pinalia maingayi (Hook.f.) Kuntze, Appendicula uncata subsp. uncata Ridl. and including two species new to science, which have been recently described: Bromheadia petuangensis Rusea \& Besi (in press in Pakistan Journal of Botany) and Pinalia domii Rusea \& Besi (unpublished). These new, rare, and endemic species were all collected and rescued from the logging sites. The logged forests were expected to have a higher likelihood harbouring rare and endemic 
TABLE 2. Comparison based on diversity and density of orchid species in DSFs of Kuala Koh, Gua Musang, Kelantan and Air Canal, Tanah Merah, Kelantan; and logging sites in Tasek Kenyir, Hulu Terengganu.

\begin{tabular}{lccccc}
\hline Studied sites & Species richness & Shannon Index, H & Simpson Index, D & Evenness, E & Density (plants $\left./ \mathbf{m}^{2}\right)$ \\
\hline DSFs & 14 & 2.47 & 0.90 & 0.94 & 0.0133 \\
\hline Logging Sites & 96 & 4.50 & 0.99 & 0.99 & 0.0040 \\
\hline
\end{tabular}

TABLE 3. Diversity of epiphytic orchids found in the logging sites of Tasek Kenyir area.

\begin{tabular}{lccccc}
\hline Studied sites & $\begin{array}{c}\text { Species } \\
\text { richness }\end{array}$ & Shannon Index, H & Simpson Index, D & Evenness, E & Density (plants $\left./ \mathbf{m}^{2}\right)$ \\
\hline Logging Sites & 94 & 4.46 & 0.99 & 0.98 & 0.0013 \\
\hline
\end{tabular}

species than the secondary forests, seeing that the area covered was larger and the fallen trees provided the chance to harvest more epiphytic orchids which are usually unreachable and difficult to see in the non-logged forests.

Diversity analyses and density.- Table 2 shows that the DSFs had a higher orchid density ( 0.0133 plants/ $\left.\mathrm{m}^{2}\right)$ when compared with the logging sites $(0.0040$ plants $\left./ \mathrm{m}^{2}\right)$ although the total of the area covered $(1,050$ $\left.\mathrm{m}^{2}\right)$ was very much lower than the latter $\left(73,550 \mathrm{~m}^{2}\right)$. However, the logged forests had a higher diversity of orchid species $(\mathrm{H}=4.50$ and $\mathrm{D}=0.99)$ compared with the secondary forests $(\mathrm{H}=2.47$ and $\mathrm{D}=0.90)$ with 94 species $(97.9 \%)$ of the orchids encountered being epiphytes. The high $\mathrm{H}$ and $\mathrm{D}$ values are also supported by the high $\mathrm{E}$ values ( $\mathrm{E}$ value of logging sites $=0.99>$ E values of DSFs $=0.94$ ) implies that the abundance of clumps or populations of each species in the logging sites was evenly distributed without being dominated by only one species.

The logging sites were dominated by epiphytic orchids which were found attached to the fallen trees within a $73,550 \mathrm{~m}^{2}$ area with a total density of 0.0013 plants $/ \mathrm{m}^{2}$ (Table 3$)$. The $\mathrm{H}(\mathrm{H}=4.46)$ and $\mathrm{D}(\mathrm{D}=0.99)$ values were high indicating a high diversity of epiphytic orchids (Table 3). The high $\mathrm{E}$ value $(\mathrm{E}=0.98)$, shows that the epiphytic orchid diversity was also influenced by their evenly distributed abundance and absence of dominant species (Table 3). In comparison, according to the data shown in Table 4 the density of epiphytic orchids in Gawi (Site 2) was higher [Gawi (Site 2) = 0.0035 plants $/ \mathrm{m}^{2}>$ Gawi (Site 1) $=0.0026$ plants $/ \mathrm{m}^{2}>$ Petuang (Site 3) $=0.0010$ plants $\left./ \mathrm{m}^{2}\right]$ even though the total area studied was lower [Gawi (Site 2) $=9,500 \mathrm{~m}^{2}$
TABLE 4. Comparison on densities of epiphytic orchid species and fallen tree hosting orchid(s) between the three studied logging sites in Tasek Kenyir area.

\begin{tabular}{|c|c|c|c|}
\hline Parameters & $\begin{array}{l}\text { Gawi } \\
\text { (Site 1) }\end{array}$ & $\begin{array}{l}\text { Gawi } \\
\text { (Site 2) }\end{array}$ & $\begin{array}{l}\text { Petuang } \\
\text { (Site 3) }\end{array}$ \\
\hline Species Richness & 26 & 33 & 51 \\
\hline $\begin{array}{l}\text { Number of Fallen Trees } \\
\text { Hosting Orchid(s) } \\
\text { (plants) }\end{array}$ & 19 & 29 & 25 \\
\hline Total Area Studied $\left(\mathrm{m}^{2}\right)$ & 10,000 & 9,500 & 53,000 \\
\hline $\begin{array}{l}\text { Density of Orchids } \\
\text { Species (plants } / \mathrm{m}^{2} \text { ) }\end{array}$ & 0.0026 & 0.0035 & 0.0010 \\
\hline $\begin{array}{l}\text { Density of Fallen Trees } \\
\text { Hosting Orchid(s) } \\
\left(\text { plants } / \mathrm{m}^{2}\right)\end{array}$ & 0.0019 & 0.0031 & 0.0005 \\
\hline
\end{tabular}

$<$ Gawi (Site 1$)=10,000 \mathrm{~m}^{2}<$ Petuang $($ Site 3$)=53,000$ $\mathrm{m}^{2}$ ]. The densities of fallen trees hosting orchid(s) also demonstrates a similar pattern; Gawi (Site 2) had a higher density than the other studied sites [Gawi (Site $2)=0.0031$ plants $/ \mathrm{m}^{2}>$ Gawi (Site 1$)=0.0019$ plants $/$ $\mathrm{m}^{2}>$ Petuang $($ Site 3$)=0.0005$ plants $\left./ \mathrm{m}^{2}\right]$.

Pearson's correlation was performed to determine the correlation between the density of orchid species and density of fallen trees hosting orchid(s) in the logging sites. Table 5 shows a significant and strong positive correlation between the densities of orchid species and the densities of fallen trees hosting $\operatorname{orchid}(\mathrm{s}), \mathrm{r}=0.993, \mathrm{n}=3, \mathrm{p}=0.037$. This implies that a single logging activity in a small area could accumulate and expose a high abundance and diversity of orchids to imminent danger. 
Microclimate of the disturbed forests.- Table 6 and Figure 2 show the significant difference of the microclimate between DSFs and logging sites. The max temperature recorded in the clear logged forests $\left(34.9 \pm 1.0 \quad{ }^{\circ} \mathrm{C}\right)$ was significantly higher $[\mathrm{H}(1)=6.621, p=0.010]$ than in the DSFs with trees canopies $\left(31.1 \pm 0.3{ }^{\circ} \mathrm{C}\right)$ (Figure $2 \mathrm{~B}$ ), with mean rank of 13.2 for logging sites and 5.5 for DSFs. The high temperature recorded in logging sites was also linked to its significantly lower min values $[\mathrm{H}(1)=5.889$, $p=0.015]$ of ARH, with mean rank of 8.93 for logging sites and 16.17 for DSFs (Figure 2C). Moreover, the microclimate values on temperature for both different types of disturbed forests are also much higher than the local climate's; with mean value of Min Temperature $=23.2{ }^{\circ} \mathrm{C}$ and mean value of Max Temperature $=30.9{ }^{\circ} \mathrm{C}$ (Malaysian Meteorology Department 2016, 2017). Simultaneously, both areas would have had lower ARH if compared to the local climate with mean value of ARH is $86.8 \%$ (Malaysian Meteorology Department 2016, 2017). This could imply that the microclimate of disturbed forests was much more intense if compared to the local climate of the surrounding areas.

The SM level shows a similar pattern where the min and max values of SM recorded for logging sites were lower than DSFs, although the difference was not statistically significant due to the small sample size studied and limited accessible area in the DSFs (Figure 2G-H).

Optimum range of light intensities on orchid development was referred to Guo et al. (2012), a study tested on wild Phalaenopsis, where the range of extreme low and high light intensities are $0-1,350$ $1 \mathrm{x}$ and 17,550-24,300 $1 \mathrm{x}$, respectively (the values recorded were converted from $\mu \mathrm{mol} \mathrm{m}^{-2} \mathrm{~s}^{-1}$ to Lux unit). The range normally used in a field study is between 15,120-20,520 lx (Chen \& Wang 1996). Based on the data collected on light intensities that also defines level of illuminance, it does not inflict any injurious effects as the values were still within the adaptable range. However, the values recorded shown a slight deviation from the specified range values of the used Lux meter, where the standardized specification of the meter signifies the average outdoor sunlight normally ranged between 20,000 $\mathrm{lx}$ to 50,000 $\mathrm{lx}$. The values recorded for logging sites only reached up to the max value of $20,000 \mathrm{~lx}$, in which, the light intensity for the opened canopies forest should have been at least 20,000 lx.

TABLE 5. Correlation between number of epiphytic orchid species and the number of fallen trees hosting orchid(s) found in the logging sites of Tasek Kenyir area. *Correlation is significant at the 0.05 level (1-tailed).

Pearson's Correlation Coefficient

Density of Orchid Species

\begin{tabular}{|c|c|c|}
\hline \multirow{3}{*}{$\begin{array}{l}\text { Density of Orchid } \\
\text { Species }\end{array}$} & $\begin{array}{l}\text { Pearson } \\
\text { Correlation }\end{array}$ & 1 \\
\hline & Sig. (1-tailed) & \\
\hline & $\mathrm{N}$ & 3 \\
\hline \multirow{3}{*}{$\begin{array}{l}\text { Density of Fallen Trees } \\
\text { Hosting Orchid(s) }\end{array}$} & $\begin{array}{l}\text { Pearson } \\
\text { Correlation }\end{array}$ & $0.993^{*}$ \\
\hline & Sig. (1-tailed) & 0.037 \\
\hline & $\mathrm{N}$ & 3 \\
\hline
\end{tabular}

TABLE 6. Summary of topographic and ecological predictors (mean \pm SE) of DSFs in Kuala Koh, Gua Musang, Kelantan and Air Canal, Tanah Merah, Kelantan; and logging sites in Tasek Kenyir, Hulu Terengganu. Notes: Average rainfall recorded in the current study was between 125.6-414.9 mm (Malaysian Meteorology Department, 2016, 2017); SE: Standard error; $\Delta$ : Elevations (m a.s.1.) above sea level; ARH (1-100\%): Air Relative Humidity; SM (1-10\%): Soil Moisture; LI (lx): Light Intensity in Lux unit; Min: Minimum value; Max: Maximum value; N-value (Logging Sites) $=15 ; \mathrm{N}$-value $(\mathrm{DSFs})=6$. Different superscripts indicate significant differences $(\mathrm{p}<0.05)$ in Kruskal-Wallis one-way ANOVA with multiple pairwise comparisons.

\begin{tabular}{|c|c|c|c|c|c|}
\hline Disturbed Forests & Temperature $\left({ }^{\circ} \mathrm{C}\right)$ & $\begin{array}{c}\text { ARH } \\
(1-100 \%)\end{array}$ & 니 (Ix) & $\begin{array}{c}\text { SM } \\
(1-10 \%)\end{array}$ & $\Delta$ \\
\hline \multirow{2}{*}{$\begin{array}{l}\text { Disturbed Secondary } \\
\text { Forests (DSFs) }\end{array}$} & Min: $28.8 \pm 0.5^{a}$ & Min: $75.8 \pm 1.6^{\mathrm{a}}$ & Min: $3856.7 \pm 1193.0^{a}$ & Min: $2.2 \pm 0.3^{a}$ & \multirow{2}{*}{$44.4-129.9$} \\
\hline & Max: $31.1 \pm 0.3^{b}$ & Max: $88.0 \pm 2.4^{c}$ & Max: $17833.3 \pm 1641.5^{c}$ & Max: $3.3 \pm 0.5^{b}$ & \\
\hline \multirow{2}{*}{$\begin{array}{l}\text { Logging Sites (logged } \\
\text { forests) }\end{array}$} & Min: $31.2 \pm 1.1^{\mathrm{a}}$ & Min: $67.0 \pm 2.3^{b}$ & Min: $9180.0 \pm 1177.7^{b}$ & Min: $2.0 \pm 0.4^{a}$ & \multirow{2}{*}{$98.8-244.6$} \\
\hline & Max: $34.9 \pm 1.0^{c}$ & Max: $86.7 \pm 1.7^{c}$ & Max: $17866.7 \pm 960.5^{c}$ & Max: $3.2 \pm 0.9^{b}$ & \\
\hline
\end{tabular}




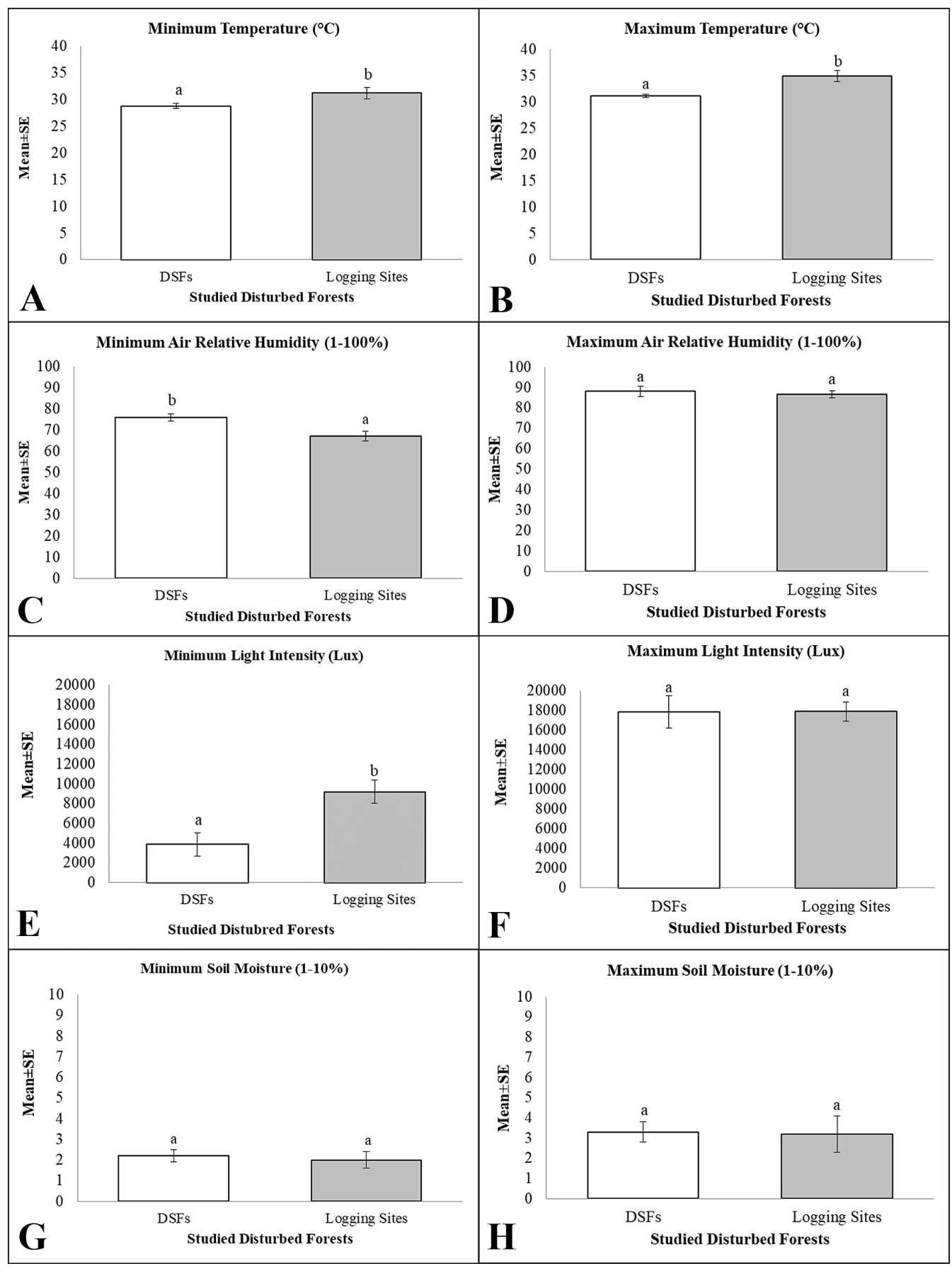

FiguRE 2. In-situ ecology parameters recorded in disturbed forests of Terengganu and Kelantan regions. A. Mean values of Minimum Temperature. B. Mean values of Maximum Temperature. C. Mean values of Minimum ARH. D. Mean values of Maximum ARH. E. Mean values of Minimum Light Intensity. F. Mean values of Maximum Light Intensity. G. Mean values of Minimum Soil Moisture. H. Mean values of Maximum Soil Moisture. Mean \pm SE is the means with standard error of the each parameter. Different superscripts indicate significant differences $(p<0.05)$ in Kruskal-Wallis one-way ANOVA with multiple pairwise comparisons. 


\section{Discussion}

Relationship between species abundance, species density and the habitat type.- The abundance of orchids and their survival in the disturbed forests were driven by the intensity of disturbance faced by each population in their particular habitat. The results show that the mildly disturbed secondary forests had a higher orchid density than the highly disturbed logging sites. This was certainly due to the ecosystem variations altered by the anthropogenic-driven disturbances. These variations were linked to the weather and microclimatic conditions, especially temperature, humidity, precipitation, and light intensity, and also the presence of supporting trees and organisms such as fungi, mosses, and pollinators.

In comparison, the secondary forests clearly had a healthier environment for orchid survival as the habitat conditions (moisture and nutrient availabilities) were still suitable for the growth of both epiphytic and terrestrial orchids. Furthermore, the presence of standing and healthy trees gave a better support for the epiphytic orchids. The trees were significant in assisting orchid photosynthesis, and also the pollination and reproduction (Cribb et al. 2003, Cozzolino \& Widmer 2005).

Studies have shown that terrestrial orchids do not survive in secondary habitats or invaded conserved forest (Williams-Linera et al. 1995, Bergman et al. 2006). However, several small populations of terrestrial Apostasia nuda R.Br. and Corymborkis veratrifolia were found in the DSFs of Kuala Koh and Tanah Merah. Despite of the heavily damaged by mudslides and heavy flooding since 2014, there are some pockets of the forests that were not affected and terrestrial orchids are still found there. The fragments of the unlogged forests are still very much protected by the forest canopy from the sun, and thus provide a tolerable environment for the orchid growth. This is where small populations of the terrestrials Calanthe ceciliae Rchb.f. and Hetaeria oblongifolia Blume were found. The low number of terrestrial orchids in the logged forests was greatly attributed to the poor soil condition, where humus-rich soil surface was eroded badly during logging that alters the $\mathrm{pH}$, humidity and the nutrients. The full exposure to sunlight and the radiation heats up the surface in the logged forest without canopy protection had distorted the atmospheric and soil microclimatic stratifications (Werner \& Gradstein 2009, Benítez et al. 2015), thus killing all the exposed epiphytic and terrestrial plants.

Small changes in ecological conditions may be deleterious to intolerant species. Even slight differences in the humidity may be significant for species near their threshold levels of water supply (Werner \& Gradstein 2009), especially epiphytes. Orchids are resistant and adaptive towards distress environmental conditions as long as atmospheric and soil moisture levels and sunlight intensity are tolerable for their survival (Hietz et al. 2006). Nevertheless, they would flourish better in less disturbed areas under the forest canopy where they can obtain optimal light, water, and nutrients to reach maturity for reproduction (López \& Runkle 2005). Therefore, it is highly recommended that the secondary forest and forest fragments left after logging are identified and protected as these areas were observed to still harbour precious orchid species.

Study based on subfamilies and growth habits.Subfamily Epidendroideae was found to be the best represented in our study areas. They are predominantly epiphytic and often have aerial roots (Holttum 1964, Seidenfaden \& Wood 1992). In the current study, only one species of this subfamily, Calanthe ceciliae, was found growing as a terrestrial in the logging sites. Compared to the other lineages, orchids from this subfamily are known to have a wide distribution area along the elevation gradients. They are always found to be abundant in extreme environments, are disturbance-resilient and exhibit more droughttolerance characteristics when compared with the other subfamilies (Rada \& Jaimez 1992, Zhang et al. 2015, Yang et al. 2016). Previous studies of degraded vegetation indicated the success of vascular epiphytes, including epiphytic orchids entirely belonging to Epidendroideae subfamily (Hietz et al. 2006, Werner \& Gradstein 2009, Huda \& Wilcock 2011). This subfamily is largely characterized as shade-loving species and only a small number are sun-loving. It might be expected that shade-demanding species would find habitat in open forests with direct exposure to the sun's rays, such as logging sites, less favourable. Although, conversely, sun-loving epiphytic orchids, such as Bromheadia Lindl. with tough stem and many small or terete leaves, should find the high light 
environment in the opened forest equally represented in the upper canopy of the closed forest (Hietz et al. 2006).

None species of Cypripedioideae and Vanilloideae subfamilies were recorded from the studied sites. Cypripedioideae is mostly terrestrial, with a few epiphytes and lithophytes; in our area, species belonging to this subfamily are highland species with coriaceous or leathery leaves, without pseudobulbs and only adapted to wet and cool conditions (Seidenfaden \& Wood 1992). Meanwhile, Vanilloideae has both warm and cool growing species, yet, have always had been recorded with lower occurrence in Peninsular Malaysia, except for Vanilla griffithii Rchb.f., a commonly found species in lowland and swamp forests (Go \& Hamzah 2008, Go et al. 2011).

Dominance of epiphytic orchids in logging sites.Epiphyte diversity tends to be reduced markedly following disturbance (Wolf 2005, Nöske et al. 2008), with variation due to the severity of disturbance and the types of disturbed habitat studied (Hietz 2005, Holz \& Gradstein 2005). Conversely, in the current study, there was a high abundance of epiphytic orchids found in the logging sites (Fig. 3).

However, this does not suggest that orchid diversity and abundance increases with the intensity of anthropogenic-driven disturbance. A high abundance of epiphytic orchids and the significant difference in densities of orchids recorded between the logging sites in Tasek Kenyir area are assumed to be correlated with the abundance or densities of the fallen trees in the logging sites, disturbance-induced dryness stresses, durations of exposure to the anthropogenicinduced disturbance (claim is made based on a survey and empirical evidence), and less favourable soil conditions for the terrestrial orchids to flourish at both sites studied.

A further study on the effect of human-induced environmental disturbance on the survival of orchid community in the degraded forests would help government agencies, conservation biologists and policy makers in formulating better conservation strategies. According to local villagers, the logging activity in Petuang had started some years (unspecified) before the logging activity in Gawi that began in 2014. Thus, time wise, it implies that the orchids in Petuang had been exposed longer to the extreme environment conditions, plus, a high number of them were found degraded with severe sun-damage and dehydration during the field collections, when compared with our collections from Gawi.

The epiphytic orchids have advanced adaptations of one or more organs to allow them to survive the long warm and dry environment conditions. Bulbophyllum and Dendrobium species for example have storage organs like pseudobulbs and pseudobulbous stems, fleshy or leathery leaves, and aerial roots with velamen as a protective layer against water loss (Zotz \& Winkler 2013, Zhang et al. 2015) and sun radiation damage (Chomicki et al. 2015), including Thrixspermum Lour, and the leafless orchids Taeniophyllum Blume that have photosynthetic roots. Unfortunately, having adaptations that allow them to survive the long warm and dry conditions do not help them to survive extended periods of desiccation since most of the specimens collected in this study exhibited dormancy and were dying upon collection.

Effects of anthropogenic-driven disturbances towards orchids abundance and survival in the disturbed forests.- The relationship between environmental conditions and orchid abundance has been frequently reported, but little is understood for orchid responses towards micro-climatic challenges and their abundance in the degraded forest with extremely fragmented tree canopies and vegetation. The canopy disruptions caused by clear-cut logging produced openings in the canopy, which had significantly affect ambient temperature and moisture. In the undisturbed-closed forest, the sun irradiation is converted into heat at the interface of the atmosphere and canopy (Werner \& Gradstein 2009). The heat intensity was more severe in disturbed-opened canopy forests or clear felling logging sites.

The microclimate of the disturbed forests

Right, FIGURE 3. Epiphytic orchids on the fallen trees and ground threatened by direct exposure to the sun irradiation and dryness in the logging sites of Tasek Kenyir area. A. Callostylis pulchella, Renanthera histrionica, Pinalia maingayi. B. Cleisostoma scortechinii. C. Coelogyne cumingii. D, E. Grammatophyllum speciosum. F, G, H. Coelogyne foerstermannii. Photos by DigitalDome. 





showed lower ARH and extremely high surrounding temperature if compared to undisturbed forested area. The direct exposure to sun radiation and heat had imposed desiccation, a state of extreme dryness, and drought-heat physiological stress towards the exposed orchid community in the forest. Deriving from a study tested on an epiphytic orchid leaves, Phalaenopsis, the optimal temperature for photosynthetic carbon fixation ranges from $29 / 25{ }^{\circ} \mathrm{C}$ to $32 / 28{ }^{\circ} \mathrm{C}$ day/night temperature (Guo \& Lee 2006, Guo et al. 2012). But, the temperature recorded for the logging sites in the current study was much higher and exceeded the optimal range required for energy production through photosynthesis. The heat irradiation caused burn and browning of the plant parts, which would eventually lead to injuries and death.

Generally, the higher the temperature, the lower the humidity or moisture, and biologically, it means the lower the turgor pressure in plant cells. The changes in turgor pressure in cells and tissues could also trigger the signal transduction pathways (Beauzamy et al. 2014) and structural proteins (Yooyongwech et al. 2008) involved in flower development: flower opening, anther dehiscence, and pollen tube growth. Thus, the exhibitions of signs of dying such as flowers or buds browning, flowers or buds dropping off (bud or bloom blast) and dormant, and pseudobulbs browning and shriveled in the disturbed forests during the field collections were most caused by high temperature (extreme heat irradiation) and extreme dryness during drought season.

Moreover, the bloom and bud blast are generally orchid's reactions to environmental shock and is the plant's way of protecting itself. This might be brought on by a sudden change in temperature and reduced water potential in plant cells to trigger the flower or bud development. By shedding its buds, the orchid can divert more energy to maintaining its vital systems (Beauzamy et al. 2014). A continuous sugar or carbohydrate supply to the apex of a reproductive bud through photosynthetic carbon fixation is essential for continued floral development (Wang 1995, Konow \& Wang 2001). Therefore, maximizing sugar production with optimal light intensity is important for orchid growth and flowering (Guo et al. 2012).

The browning observed on the leaves of orchids collected from the logging sites could be due to two possibilities: (i) reduction of the chlorophyll content which has reduced the pigment responsible for the green colour of the leaves; (ii) too high light intensity provokes an increase in leaf temperature causing photosynthetic machinery degradation in plant cells (Edmond et al. 1978, Sinha \& Häder 2002, Stancato et al. 2002, Chomicki et al. 2015).

The deviations occurred to the data collected in the current study for light intensities could be related to various reasons, including time of the day, season, geographic location, weather, or device systematic error. The light intensity could be also affected by dust particles and atmospheric water vapor, slope and elevation (Edmond et al. 1978). The units Lux is simply based on visual sensitivity and do not provide information on the energy or photon content of light, which truly influences the photosynthesis or sugar production in the leaf. Hence, in order to understand better the light intensity for a study relating to plant responses, the suitable unit is the $\mu \mathrm{mol} \mathrm{m}-2 \mathrm{~s}-1$ [number of photons received per unit time (s) on a unit area (m)]. Similarly, the effects of ultraviolet radiation (UV-radiation) on orchids in wide-opened canopy forest are another perspective recommended for future studies of orchids in disturbed forest. UV-radiation is a major hazard for living organisms exposed to sunlight, causing DNA mutation, and plasma membrane lipid peroxidation.

A small number of terrestrial orchids encountered in this study were suspected to be influenced by the soil properties and ground conditions. Inconclusively, aside from the massive clearance of ground vegetation by tractor, the low values of soil moisture (SM) and absence of buffering trees and leaf litter were predicted to reflect and influence the geographical distribution of the individual terrestrial orchids in the logged forests and DSFs. Soil moisture was included as the predictor, because, even though moisture affected by soilforming factors (mineralization and holding capacity), moisture potentially is a strong factor controls other soil properties, including $\mathrm{pH}$ and nutrient (Pulla et al. 2016). Although, the min and max values of SM recorded for both sites were not statistically significant different, nonetheless, in comparison, it appears that the min moisture values recorded for logging sites were lower than the moisture values recorded in a previous study on soil properties associated with dry forest 
community (see Pulla et al. 2016). This suggests that the dryness stress faced by the orchids community in the logging sites was much severe than in the naturally occurred dry forest.

The surface erosion occurred in the logging sites as a result of absence of buffering trees and leaf litter, and direct exposure of mineral soil to the impact of rain might have reduced the soil holding capacity which would have also reduced the soil moisture and nutrient (Raghubanshi 1992, Li et al. 2014), and compromising the orchid growth and their mycorrhizal association (Batty et al. 2001, Harrington \& Schoenholtz 2010, Li et al. 2014). Increased frequency of erosions and direct heat exposure would affect the microorganism community, too. In nature, primarily, the terrestrial orchids also require the mycorrhizal association (fungal-roots) for survival and nitrogen fixation (Brundrett 2002). For a better understanding, it is desirable to include soil $\mathrm{pH}$ in future studies for a better clarification and to determine the relationship between level of mineralization and nitrification of soil and orchid distribution in the disturbed forest.

Conclusions. Our results show a high diversity of orchids found at Peninsular Malaysia's disturbed forest areas. The diversity indexes reflect a greater orchid diversity in the areas with wide-opened canopies of the logged forests than in the canopy-covered DSFs, with several rare species and six species being endemic to Peninsular Malaysia, including two new species to science, and of these, largely were epiphytes, typically with robust morphological appearance and adaptations. This high species diversity and indexes in wide-opened canopies logged forests is due to accessibility of epiphytic orchids on fallen trees. The current study covered only $5 \%$ of the total area of the disturbed forests in Terengganu and Kelantan through
3 years' botanical collections. We anticipate much more orchids at risk could be found in the logged forest following the positive correlation between the density of epiphytic orchids and the density of fallen trees in the logging sites. This orchid community appears to be critically endangered due to ongoing logging activities in the region. The canopy disruptions caused by forest logging and the mudslides had reduced the quality of the orchid habitats. The anthropogenic-driven disturbances had harmfully altered the humidity and temperature in the affected areas. The disturbance-induced drought stress had damaged the orchid morphologically and interfered with their phenology. This study has resulted in new hope for rapid orchid species documentation endeavour in Malaysia, as many epiphytic orchids are accessible resulting in many new discoveries of new records and species to science.

ACKNowledgements. We would like to extend our appreciation to the Forest Department Peninsular Malaysia and Department of Wildlife and National Parks Peninsular Malaysia for granting us the permit [Reference No. JH/100 Jld. 16(14)] to access the studied areas and to the UPM-KRIBB (Korea Research Institute of Bioscience and Biotechnology) Vot. 6384300, and Universiti Putra Malaysia for the research funding (Putra Grant No. 9413603), and Yayasan Biasiswa Tunku Abdul Rahman (YBSTAR) scheme for sponsoring this Master of Science degree undertaking. The authors are also very thankful to the staff and friends in the department for their support and invaluable assistance throughout this project. We owe our sincere thanks to many experts for their opinions and helps on the identifications; Jeffrey Champion (Bedugul Tabanan, Bali, Indonesia), Jim Cootes (Centre for Plant Biodiversity Research, Canberra, Australia), and Linus Gokusing (Kipandi Park, Malaysia).

\section{Literature Cited}

Abdullah, R., Ibrahim, A., Simin, M. H. A., Ramle, N. H., \& Rasat, M. S. M. (2017). Pemuliharaan hutan dalam kalangan masyarakat Semaq Beri di Negeri Terengganu, Malaysia (Forest conservation and the Semaq Beri community of Terengganu, Malaysia). GeografiaMalaysian Journal of Society and Space, 10(3), 113-124. [Note: Article published in Malay language]

Batty, A. L., Dixon, K. W., Brundrett, M. \& Sivasithamparam,
K. (2001). Constraints to symbiotic germination of terrestrial orchid seed in a mediterranean bushland. New Phytologist, 152(3), 511-520.

Benítez, Á., Prieto, M. \& Aragón, G. (2015). Large trees and dense canopies: key factors for maintaining high epiphytic diversity on trunk bases (bryophytes and lichens) in tropical montane forests. Forestry: An International Journal of Forest Research, 88(5), 521-527. 
Beauzamy, L., Nakayama, N. \& Boudaoud, A. (2014). Flowers under pressure: ins and outs of turgor regulation in development. Annals of botany, 114(7), 1517-1533.

Bergman, E., Ackerman, J. D., Thompson, J. \& Zimmerman, J. K. (2006). Land-use history affects the distribution of the saprophytic orchid Wullschlaegelia calcarata in Puerto Rico's Tabonuco Forest 1. Biotropica, 38(4), 492-499.

Besi, E. E., Dome, N., Mustafa, M. \& Go, R. (in press). A new species of Bromheadia Sect. Aporodes (Orchidaceae) rescued from a logging site in Terengganu, Peninsular Malaysia. Pakistan Journal of Botany.

Bridson, D. \& Forman, L. (2000). The herbarium handbook, 3rd edition. London, United Kingdom: Royal Botanic Gardens, Kew. 348 pp.

Brundrett, M. C. (2002). Coevolution of roots and mycorrhizas of land plants. New phytologist, 154(2), 275-304.

Chen, W. H. \& Wang, Y. T. (1996). Phalaenopsis orchid culture. Taiwan Sugar, 43, 6-11.

Chomicki, G., Bidel, L. P. R., Ming, F., Coiro, M., Zhang, X., Wang, Y., Baissac, Y., Jay-Allemand, C. \& Renner, S. S. (2015). The velamen protects photosynthetic orchid roots against UV-B damage, and a large dated phylogeny implies multiple gains and losses of this function during the Cenozoic. New Phytologist, 205(3), 1330-1341.

Comber, J. B. (2001). Orchids of Sumatra. Natural History Publications (Borneo) Sdn. Bhd., Kota Kinabalu, Sabah, Malaysia. 1026 pp.

Cozzolino, S. \& Widmer, A. (2005). Orchid diversity: an evolutionary consequence of deception? Trends in Ecology \& Evolution, 20(9), 487-494.

Cribb, P. J., Kell, S. P., Dixon, K. W. \& Barrett, R. L. (2003). Orchid Conservation: a global perspective. In: K. W. Dixon, S. P. Kell, R. L. Barrett \& P. J. Cribb (Eds), Orchid conservation. Kota Kinabalu, Sabah, Malaysia: Natural History Publications. 1-24 pp.

Dimmit, M. (2000). Endangered Bromeliaceae. In: D. H. Benzing (ed). Bromeliaceae: profile of an adaptive radiation (pp. 609-620). Cambridge, United Kingdom: Cambridge University Press.

Edmond, J. B., Senn, T. L., Andrews, F. S. \& Halfacre, R. G. (1978). Fundamentals of horticulture (4th ed.). New York, U.S.A.: McGraw-Hill, Inc. 109-130 pp.

Fatanah, N. (2009). BatekHep: manifestasi alam dan kebergantungan hidup terhadap rimba. (Doctoral dissertation). Institut Alam Sekitar dan Pembangunan (LESTARI), Universiti Kebangsaan Malaysia, Bangi, Malaysia. [Note: Article published in Malay language]

Go, R. \& Hamzah, K. A. (2008). Orchids of peat swamp forests in Peninsular Malaysia. Forest Research Institute Malaysia (FRIM), Serdang, Malaysia. Peat Swamp Forest Technical Series No. 13. 136 pp.

Go, R., Eng, K. H., Mustafa, M., Abdullah, J. O., Naruddin, A.
A., Lee, N. S., Lee, C. S., Eum, S. M., Park, K. W. \& Choi, K. (2011). An assessment of orchids' diversity in Penang Hill, Penang, Malaysia after 115 years. Biodiversity and Conservation, 20(10), 2263-2272.

Go, R., Yong, W. S. Y., Joanes, U. \& Salleh, R. (2010). Orchids of Perlis, jewels of the forest. Revised Edition. Kuala Lumpur, Malaysia: Jabatan Perhutanan Perlis and Universiti Putra Malaysia. 152 pp.

Guo, W. J. \& Lee, N. (2006). Effect of leaf and plant age, and day/night temperature on net $\mathrm{CO} 2$ uptake in Phalaenopsis amabilis var. formosa. Journal of the American Society for Horticultural Science, 131(3), 320-326.

Guo, W. J., Lin, Y. Z. \& Lee, N. (2012). Photosynthetic light requirements and effects of low irradiance and daylength on Phalaenopsis amabilis. Journal of the American Society for Horticultural Science, 137(6), 465-472.

Gradstein, S. R. (2008). Epiphytes of tropical montane forestsimpact of deforestation and climate change. Göttingen Centre for Biodiversity and Ecology. Biodiversity and Ecology Series, 2, 51-65.

Hairul, N. M. K, Ramle, A., Mohamad, H. A. S. \& Asmawi, I. (2016). Kelestarian alam sekitar taman negara Tasik Kenyir dan impak kesejahteraan kepada masyarakat orang asli sekitar. International Conference on Education and Regional Development 2016 (ICERD 2016). [Note: Article published in Malay language]

Harrington, T. B. \& Schoenholtz, S. H. (2010). Effects of logging debris treatments on five-year development of competing vegetation and planted Douglas-fir. Canadian Journal of Forest Research, 40(3), 500-510.

Help, C. (1974). A new index measuring evenness. Journal of the Marine Biological Association of the United Kingdom, 54(3), 555-557.

Hew, C. S., Arditti, J. \& Lin, W. S. (1997). Orchid cut-flower production in ASEAN countries. In: J. Arditti (Ed.). Orchid Biology: reviews and perspectives, Volume 6 (pp. 363-401). New York, U.S.A.: John Wiley \& Sons.

Hietz, P. (2005). Conservation of vascular epiphyte diversity in Mexican coffee plantations. Conservation Biology, 19(2), 391-399.

Hietz, P., Buchberger, G. \& Winkler, M. (2006). Effect of forest disturbance on abundance and distribution of epiphytic bromeliads and orchids. Ecotropica, 12(2), 103-112.

Holttum, R. E. (1964). A revised flora of Malaya, volume 1. Orchids of Malaya. Singapore: Government Printing Office. 759 pp.

Holz, I. \& Gradstein, R. S. (2005). Cryptogamic epiphytes in primary and recovering upper montane oak forests of Costa Rica-species richness, community composition and ecology. Plant Ecology, 178(1), 89-109.

Huda, M. K. \& Wilcock, C. C. (2011). Colonisation and diversity of epiphytic orchids on trees in disturbed 
and undisturbed forests in the Asian tropics. Gardens, Bulletin Singapore, 63(1-2), 341-356.

Jaafar, N., Abdul Rasid, J., Zakaria, R., Chu Abdullah, M. R. \& Omar, M. (2007). Wild orchids of Terengganu. Terengganu, Malaysia: Forestry Department of Terengganu. 120 pp. ISBN: 978-43120-1-5.

Kiew, R., Chung, R. C. K., Saw, L. G. \& Soepadmo, E. (2010). Seed Plant Families in Peninsular Malaysia. In: R. Kiew, R. C. K. Chung, L. G. Saw, E. Soepadmo \& P. C. Boyce (Eds.), Flora of Peninsular Malaysia, Series II: Seed Plants, Volume 1 (pp 3-20). Kepong, Kuala Lumpur, Malaysia: Forest Research Institute Malaysia (FRIM).

Konow, E. A. \& Wang, Y. T. (2001). Irradiance levels affect in vitro and greenhouse growth, flowering, and photosynthetic behavior of a hybrid Phalaenopsis orchid. Journal of the American Society for Horticultural Science, 126(5), 531-536.

Larson, R. J. (1992). Population dynamics of Encyclia tampensis in Florida. Selbyana, 13, 50-56.

Li, X., Niu, J. \& Xie, B. (2014). The effect of leaf litter cover on surface runoff and soil erosion in Northern China. PLoS ONE, 9(9), e107789.

López, R. G. \& Runkle, E. S. (2005). Environmental physiology of growth and flowering of orchids. HortScience, 40(7), 1969-1973.

Lye, T. P. (2005). Changing pathways: forest degradation and the Batek of Pahang. Strategy Information Research Development, Petaling Jaya, Selangor, Malaysia. 227 pp.

MacDonald, G. M. (2003). Biogeography: space, time, and life. New York, U.S.A.: John Wiley and Sons, Inc. 528 pp.

Malaysian Meteorological Department (2016). Data on Monthly Rainfall Amount and Number of Raindays, Monthly Mean Minimum and Maximum Temperature, and Monthly mean relative humidity for Pengkalan Gawi, Terengganu. Received on August 8, 2017.

Malaysian Meteorological Department (2017). Data on Monthly Rainfall Amount and Number of Raindays, Monthly Mean Minimum and Maximum Temperature, and Monthly mean relative humidity for Pengkalan Gawi, Terengganu. Received on August 8, 2017.

Margalef, R. (1972). Homage to Evelyn Hutchinson, or why is there an upper limit to diversity. Transactions of the Connecticut Academy of Arts and Sciences, 44, 211-235.

Mondragon, D. \& Calvo-Irabien, L. M. (2006). Seed dispersal and germination of the epiphyte Tillandsia brachycaulos (Bromeliaceae) in a Tropical Dry Forest, Mexico. The Southwestern Naturalist, 51(4), 462-470.

National Herbarium of the Netherlands (NHN) (2017). Accessed on July 11, 2017 through Browse Dutch Natural History Collections: BioPortal (Naturalis) at http://bioportal.naturalis.nl/
Nöske, N. M., Hilt, N., Werner, F. A., Brehm, G., Fiedler, K., Sipman, H. J. \& Gradstein, S. R. (2008). Disturbance effects on diversity of epiphytes and moths in a montane forest in Ecuador. Basic and Applied Ecology, 9(1), 4-12.

Ong, P. T., O’Bryne, P., Saw, L. G. \& Chung, R. C. K. (2017). Checklist of orchids of Peninsular Malaysia. Perpustakaan Negara Malaysia, Kuala Lumpur, Malaysia. Research Pamphlet. No. 136. 169 pp.

Ong, P. T., O’Bryne, P., Yong, W. S. Y. \& Saw, L. G. (2011). Wild orchids of Peninsular Malaysia. Malaysia: Forest Research Institute Malaysia (FRIM) and MPH Group Publishing Sdn. Bhd. 196 pp.

Pant, B. (2013). Medicinal orchids and their uses: Tissue culture a potential alternative for conservation. African Journal of plant science, 7(10), 448-467.

Pielou, E. C. (1966). The measurement of diversity in different types of biological collections. Journal of Theoretical Biology, 13, 131-44.

Pulla, S., Riotte, J., Suresh, H. S., Dattaraja, H. S. \& Sukumar, R. (2016). Controls of soil spatial variability in a dry tropical forest. PLoS ONE, 11(4), e0153212.

Rada, F. \& Jaimez, R. (1992). Comparative ecophysiology and anatomy of terrestrial and epiphytic Anthurium bredemeyeri Schott in a tropical Andean cloud forest. Journal of Experimental Botany, 43(5), 723-727.

Raghubanshi, A. S. (1992). Effect of topography on selected soil properties and nitrogen mineralization in a dry tropical forest. Soil Biology and Biochemistry, 24(2), 145-150.

Ramle, A. (1993). Semaq Beri: komuniti orang asli di Terengganu. Kolej Agama Sultan Zainal Abidin, Terengganu, Malaysia. [Note: Article published in Malay language]

Ramle, A., Asmawi, I., Mohamad Hafis, A. S., Nur Hafizah, R. \& Mohd Sukhairi, M. R. (2014). Pemuliharaan hutan dalam kalangan masyarakat Semaq Beri di Negeri Terengganu, Malaysia. GEOGRAFIA OnlineTM Malaysian Journal of Society and Space, 10(3), 113-124. [Note: Article published in Malay language]

Rauh, W. (1992). Are Tillandsias endangered plants? Selbyana, 13,138-139.

Seidenfaden, G. \& Wood, J. J. (1992). The orchids of Peninsular Malaysia and Singapore. London, United Kingdom: Royal Botanic Garden, Kew. 779 pp.

Singapore Herbarium Online Database (SING) (2018). Accessed on January 12, 2018 through http://herbaria. plants.ox.ac.uk/bol/sing

Sinha, R. P. \& Häder, D. P. (2002). UV-induced DNA damage and repair: a review. Photochemical and photobiological Sciences, 1(4), 225-236.

Stancato, G. C, Mazzafera, P. \& Buckeridge, M. S. (2002). Effects of light stress on the growth of the epiphytic orchid Cattleya forbesii Lindl. x Laelia tenebrosa Rolfe. 
Brazilian Journal of Botany, 25(2), 229-235.

Swiss Orchid Foundation at the Herbarium Jany Renz. (2017) Accessed on July 7, 2017 through https://orchid.unibas. ch/index.php/en/database-search/advanced-search

Tuck-Po, L. (2000). Forest, Bateks, and degradation. Japanese Journal of Southeast Asian Studies, 38(2), 165-184.

Turner, I. M. (1995). A catalogue of the vascular Plants of Malaya: Orchidaceae. The Garden's Bulletin, 47(1), 559-620.

Wan Mohd Shukri, W. A., Ismail, H., Nur Hajar, Z. S. \& Mohd Rizal, S. (2007). Logged-over forests under the selective management system: a case study in Jengai Forest Reserve, Terengganu (pp 68-77). In: N. M. Nik Zanariah, K. A. Sarifah \& H. Nor Azman (Eds), Highlight of FRIM'S IRPA Projects 2006. Kepong, Malaysia: Forest Research Institute Malaysia.

Wang, Y. T. (1995). Phalaenopsis orchid light requirement during the induction of spiking. HortScience, 30, 59-61.

WCSP (2018). World Checklist of Selected Plant Families. Facilitated by the Royal Botanic Gardens, Kew. Accessed on December 28, 2018 through http://apps. kew.org/wcsp/home

Werner, F. A. \& Gradstein, S. R. (2009). Diversity of dry forest epiphytes along a gradient of human disturbance in the tropical Andes. Journal of Vegetation
Science, 20(1), 59-68.

Williams-Linera, G., Sosa, V. \& Platas, T. (1995). The fate of epiphytic orchids after fragmentation of a Mexican cloud forest. Selbyana, 16(1), 36-40.

Wolf, J. H. (2005). The response of epiphytes to anthropogenic disturbance of pine-oak forests in the highlands of Chiapas, Mexico. Forest ecology and management, 212(1-3), 376-393.

Yang, S. J., Sun, M., Yang, Q. Y., Ma, R. Y., Zhang, J. L. \& Zhang, S. B. (2016). Two strategies by epiphytic orchids for maintaining water balance: thick cuticles in leaves and water storage in pseudobulbs. AoB PLANTS, 8, plw046.

Yooyongwech, S., Horigane, A., Yoshida, M., Yamaguchi, A., Sekozawa, Y., Sugaya, S. \& Gemma, H. (2008). Changes in aquaporin gene expression and magnetic resonance imaging of water status in peach tree flower buds during dormancy. Physiologia Plantarum, 134, 522-533.

Zhang, S. B., Dai, Y., Hao, G. Y., Li, J. W., Fu, X. W. \& Zhang, J. L. (2015). Differentiation of water-related traits in terrestrial and epiphytic Cymbidium species. Frontiers in Plant Science, 6, 260.

Zotz, G. \& Winkler, U. (2013). Aerial roots of epiphytic orchids: the velamen radicum and its role in water and nutrient uptake. Oecologia, 171, 733-741. 